\title{
DEVELOPMENT OF TECHNOLOGY FOR IMPROVING PRODUCTIVITY AND QUALITY OF INJECTION MOLDING
}

\author{
Hong-Seok Park \& Xuan-Phuong Dang
}
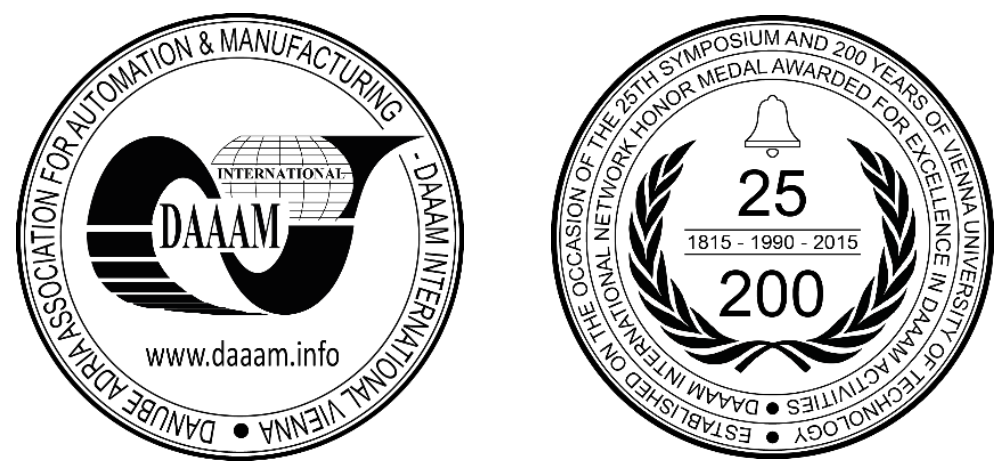

This Publication has to be referred as: Park, H[ong] S[eok] \& Dang, X[uan-Phuong] (2017). Development of Technology for Improving Productivity and Quality of Injection Molding, Proceedings of the 28th DAAAM International Symposium, pp.0309-0314, B. Katalinic (Ed.), Published by DAAAM International, ISBN 978-3-902734-11-2, ISSN 1726-9679, Vienna, Austria

DOI: $10.2507 / 28$ th.daaam.proceedings.042

\begin{abstract}
This work presents a study on the application of 3D printing technology and the smart control for improving the productivity and quality in the injection molding. The cooling time and cycle time are greatly reduced using the conformal cooling channels made by metal 3D printing technology. The geometry of the cooling channels is optimized so that the cooling efficiency increases and the aesthetic of the molded part is also improved. A case study of molding of an automotive door module is applied to verified conformal cooling technology. The research result shows that the cooling time is reduced around $23 \%$. Besides the application of 3D printing technology, a method that ensures the quality consistency of the molded parts regardless of the change of injection molding machine or the disturbance of uncertainty molding condition is proposed. The cavity sensors including pressure and temperature sensors are used to monitor the process condition inside the mold. A control strategy and an intelligent algorithms are figured out to compensate the variation of the molding condition in the mold cavity so that the quality consistency is always satisfied. This work is intended as a contribution to the development of the smart molding technology.
\end{abstract}

Keywords: smart injection molding; conformal cooling channels; cooling efficiency; 3D printing

\section{Introduction}

Injection molding is a popular manufacturing technology for making plastic products. The injection molding process includes three significant stages: filling and packing stage, cooling stage, and ejection stage. Among these stages, cooling stage is very important one because it mainly affects the productivity and molding quality. An appropriate design of cooling channel reduces cooling time, increases the productivity, and minimizes undesired defects such as sink marks, differential shrinkage, thermal residual stress and warpage. Simple and traditional cooling channels cannot result in an even and effective cooling performance when the geometry of the molded part is complex. Conformal cooling channels made by 3D printing method is one of the solutions for cooling complex part [1].

Conformal cooling channel has drawn great attention to mold designer and plastic molding researcher. Ferreira and Mateus [2] studied on rapid soft tooling with conformal cooling channels for plastic injection molding. Wang et al. [3] introduced an approach to generate spiral channels for conformal cooling system and concluded that the cooling channels derived from spiral curves introduce nearly no reduction of the rate of coolant flow. In literature, there are four types of 
conformal cooling channels: spiral conformal cooling channels [3, 4], zigzag-type conformal cooling channels [4], scaffold type conformal cooling channels [5], and Voronoi diagram type [6]. Each type of conformal channel is suitable for a specific case according to the geometry of the molded part and the decision of the mold designer.

Because of the advancement in metal 3D printing technology, the application of conformal cooling channels made by solid freeform fabrication has been becoming popular recently. The conformal cooling channels system is recognized as one of the best solutions for reducing cycle time, differential shrinkage, and warpage defects on molded parts [7,8]. Jahan and El-Mounayri [9] studied on the way of optimization of cooling channels parameters for simple cylindrical shape using simulation and design of experiment. Various researches [9-13] developed the optimal design for cooling channels that can increase the effectiveness of cooling system in the injection mold; however, the optimal design of the cooling channels in the mold has been restricted by the relatively simple cooling channel configurations. The complex molded part requires a complicated cooling channel design than simple mold. In addition, how to design the best conformal cooling channel that meets the desired requirement has not been address intensively.

The quality of the molded part mainly depends on the skill of the molding technician. Plasticization, mold filling, and solidification cooling are invisible processes which take place inside electrical mechanical components such as the heat barrel, nozzle, and mold [14]. Commonly, injection molding parameter selection and adjustments were based primarily on the experience of the operator. This approach has the risk of unanticipated outcomes such as short shot, large warpage or sink mark. Also, the quality of molded part is inconsistent shot to shot. The technician only gets feedback about the molding quality after the molded part come out of the injection machine. This means that the only way for the technician to determine the cause of any molding faults is to check the molded part after they are produced and then adjust the molding parameters for the next cycle until an acceptable product is produced. The incorporation of statistics and the use of design of experiments can improve the process control setting, but we still didn't know what is happening in the mold cavity during injection. Therefore, it is necessary to adopt a real-time smart system that monitors, records, and controls temperature and pressure signals generated by in-cavity sensors. The system is integrated with the molding machine control system. Each molding cycle initiates an accumulation of data which is stored and refreshed at the start of the next cycle [15].
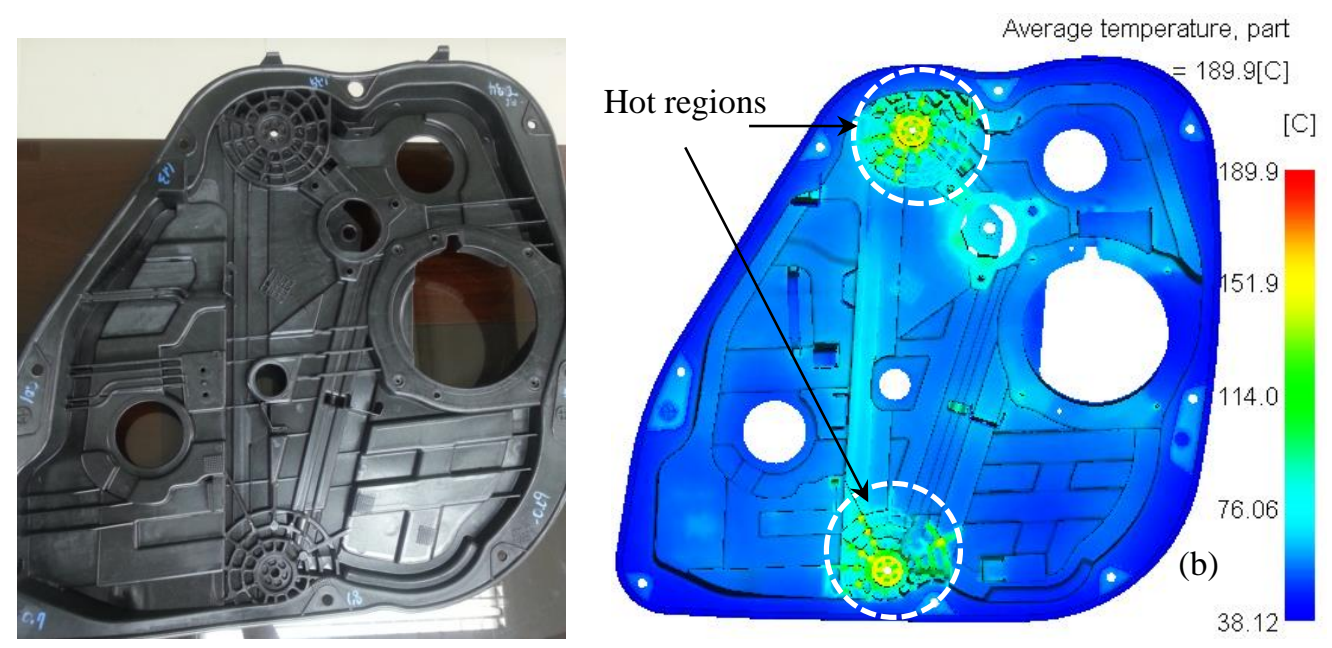

Fig. 1. The plastic door module (for automotive component) and the temperature distribution of the molded part

In this work, we solve a practical problem in injection molding industry. A complicated plastic car door module as shown in Figure 1 has been manufacturing. Due to the functionality of the molded part, the thickness of the molded part is varied. Especially, the thickness in the two regions with rib (web region) is largest. Therefore, the cooling time is longest compared to other regions. As the result, the total cycle time is long. Thus, the product producer wants to reduce the cycle time. In addition, they also want to ensure the quality and productivity regardless of the variation of process conditions. Therefore, it is necessary to develop a monitoring and the intelligent control system that can automatically find the optimal process parameters and produce a consistent product quality.

\section{The systematic research procedure}

To solve the previously mentioned problem, a systematic research procedure including six steps has been figured out as shown in Fig. 2. After analysing the problem and the development goal (reduce the cooling time for improving the productivity), we determined the appropriate cooling channel type and then designed and optimized of cooling channels by analytical method. Next, the numerical simulation was done for verification because the analytical method is based on semi-practical formulas. After design and simulation, we fabricated the cooling system with 3D printing technology and then performed the test on the real injection molding machine. The important steps in the research procedure for Improvement of cooling efficiency of injection molding are described in the next sections. 


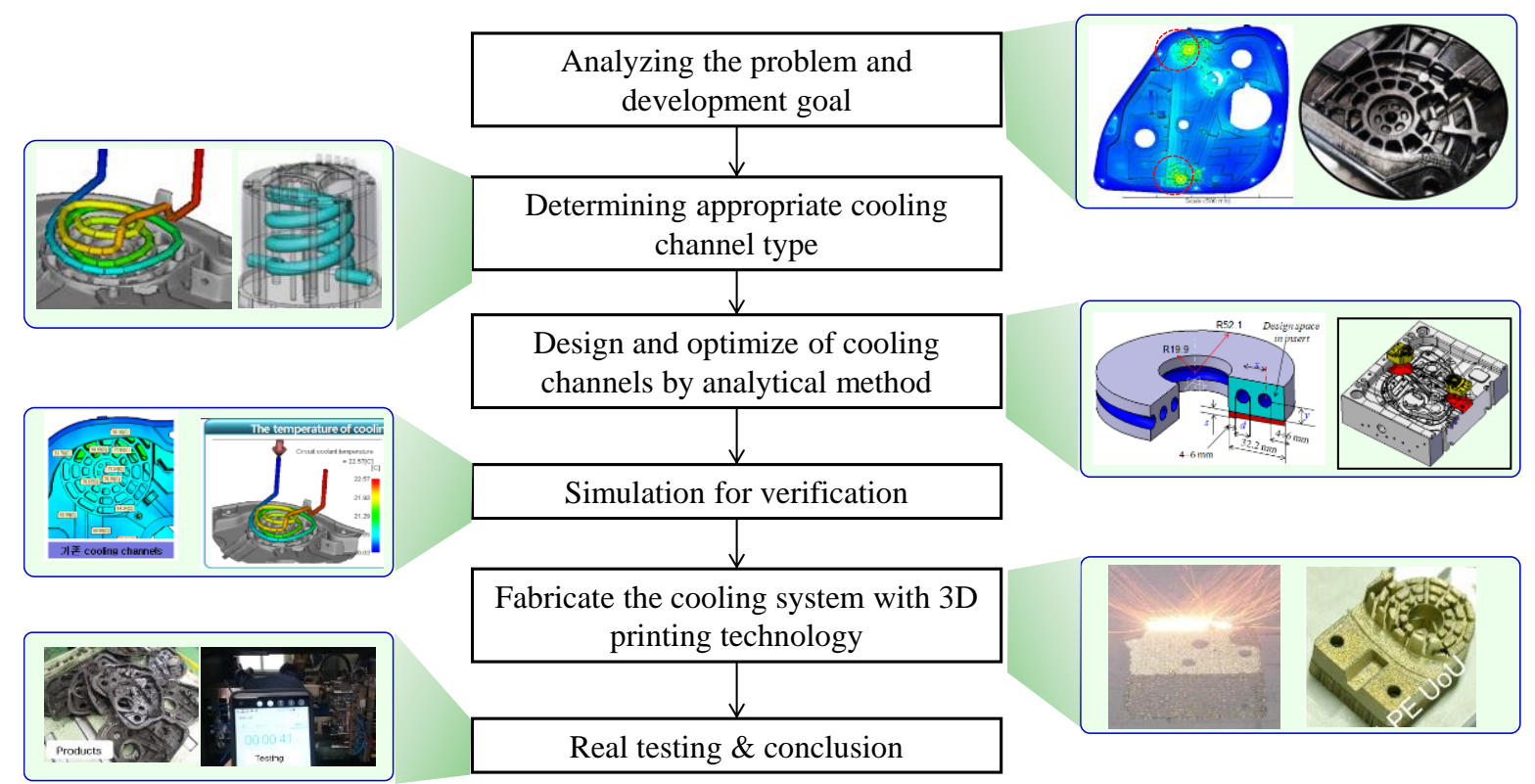

Fig. 2. The research procedure

\section{Improvement of cooling efficiency of the injection mold by $3 D$ printing technology}

\subsection{Analytical calculation}

The conformal cooling channel with the spiral form was selected as the proper cooling channel for previously mentioned injection mold. It is difficult to determine the cooling channel configuration by intuition of the designer or by the iteration of simulation. The spiral cooling channel can be modelled as a simplified equivalent model that can use the analytical method. In this work, we use the semi-practical formula to determine the optimal cooling channel configuration. The configuration including pitch $x$, depth $y$ and diameter $d$ can be derived by the equation [16]:

$$
\frac{\left[c_{p}\left(T_{M}-T_{E}\right)+i_{m}\right] \rho \frac{s}{2} x}{T_{W}-T_{C}}\left\{\frac{1}{2 \pi k_{s t}} \ln \left[\frac{2 x \sinh \left(2 \pi \frac{y}{x}\right)}{\pi d}\right]+\frac{1}{0.03139 \pi R_{e}^{0.8}}\right\}=\frac{s^{2}}{\pi^{2} a} \ln \left[\frac{4}{\pi}\left(\frac{T_{M}-T_{W}}{T_{E}-T_{W}}\right)\right]
$$

where $a=\frac{k_{p}}{\rho c_{p}}$ and Reynolds number $R_{e}=u \frac{d}{v}$. The nomenclature of other parameters is listed in Table 1 .

$T_{E}, T_{M}, T_{W}, T_{C}, k_{s t}, s$ are ejection temperature, melt temperature, mold wall temperature, temperature of water (coolant), thermal conductivity of mold, molded part thickness, respectively.

It can be seen that the equation (1) shows the relation among cooling time and geometrical of cooling channel.

Mathematically, with pre-set $T_{M}, T_{E}, T_{W}, T_{C}$, predefined thermal properties of material, the equation (1) presents the relation between cooling time $t_{c}$ and the variables related to cooling channels configuration including pitch $x$, depth $y$ and diameter $d$.

Solving the equation (1) without constraints, multi solutions are obtained. However, due to the constraint $32.2-6 \times 2$ $\leq \mathrm{x}+\mathrm{d} \leq 32.2-5 \times 2$ of the design space, the diameter and the pitch of the cooling channel are selected as 7.0 and $13.9 \mathrm{~mm}$, respectively. We used the analytical results to determine the geometry of cooling channel. This data was then used to model the cooling channels for numerical simulation. If the target time to reach the ejection temperature is not satisfied, the distance from the cooling channels to the mold surface is fine-tuned and additional simulations are carried out until the satisfaction is obtained.

\subsection{Design and simulation of conformal cooling channels}

The conformal cooling system has to be designed based on the current mold with conventional cooling channels. Because the mold is large and the molded part is mainly in flat shape, the main cooling system of the old mold is retained. Two inserts with conformal cooling channels are added into the mold core (Fig. 4).

To analyse the thermal performance of the original mold as well as to develop the new cooling system, we used the simulation tool (Moldflow software). The steady-state cool simulation was used to analyze both the temperature of the part and cycle averaged temperature distribution in the mold. The diameter, pitch, and depth of the cooling channels for building the simulation model were obtained from analytical method. 

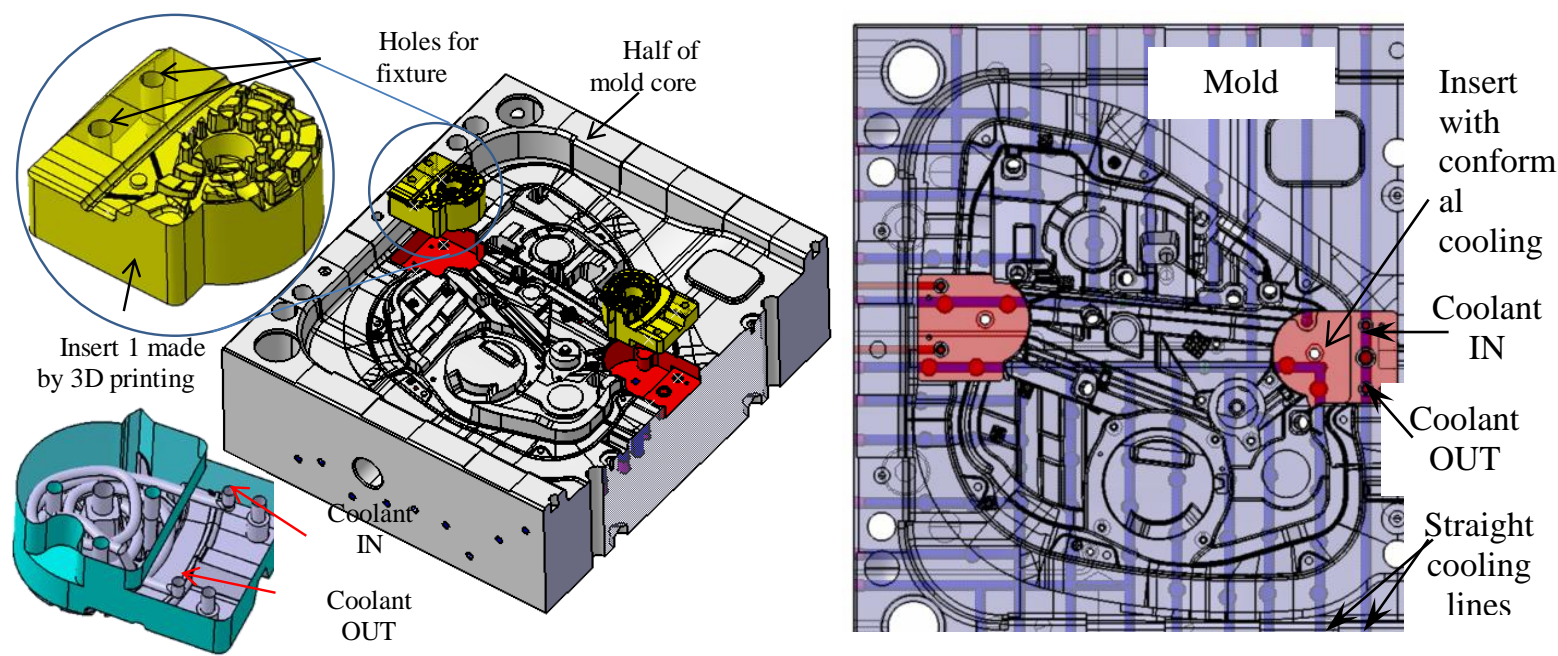

Fig. 3. The layout, structure and assembly of insert with conformal cooling channels in the mold

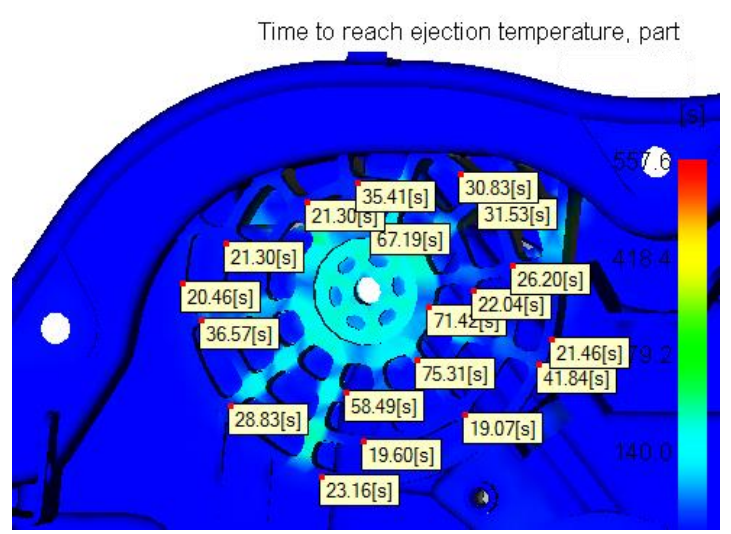

(a) Conventional cooling channel

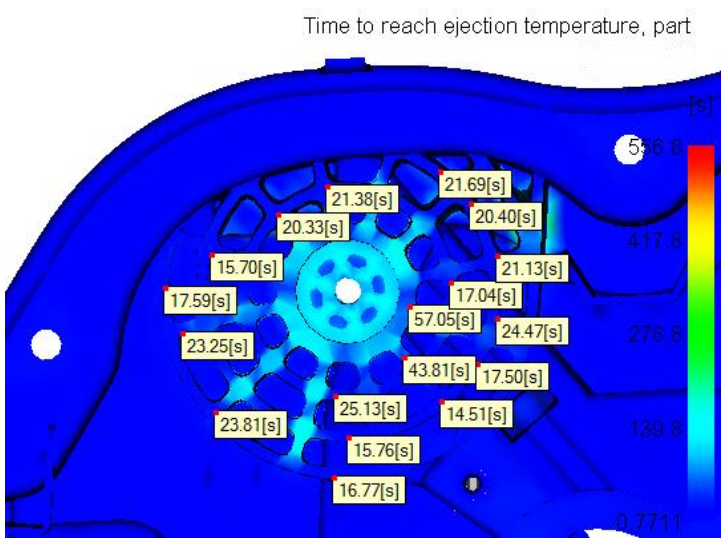

(b) Conformal cooling channel

Fig. 4. Comparison of time to reach the ejection temperature between conformal and conventional cooling channels

The simulation results show that the conformal cooling channels give more even temperature distribution. The temperature in the hot regions (Fig. 1) is reduced significantly (around $23 \div 28{ }^{\circ} \mathrm{C}$ depend on the position). The time to reach ejection temperature of molded par is reduced about (23.14-33.57)/33.57 $=31 \%$ (when 18 sample points are taken into account as shown in Fig. 5, the average time to reach ejection temperature of conventional and conformal cooling channels are 33.57 and $23.14{ }^{\circ} \mathrm{C}$, respectively). This means that the cooling time can be reduced up to $31 \%$.

\subsection{Fabrication of inserts with conformal cooling channels using metal 3D printing technology}

After design and simulation, the two insets were fabricated by selective laser melting method on MetalSys 150 3D printer with powder material that has composition as HP 4A(1). One of the insert is shown in Figure 6. The two inserts were machined and assembled to the mold for testing their cooling performance.

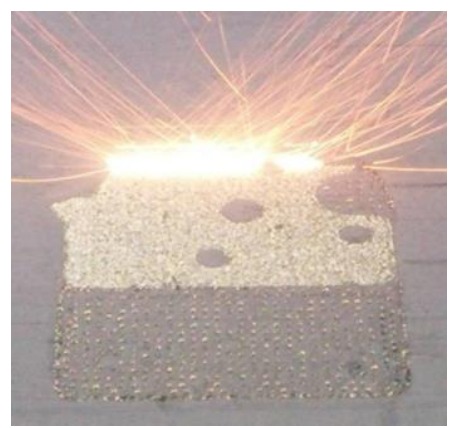

(a) Printing process

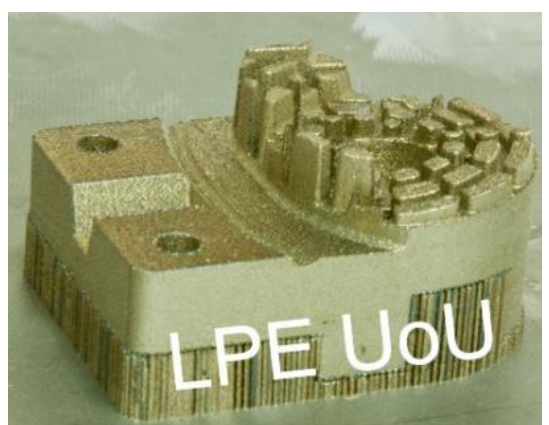

(b) Insert after printing

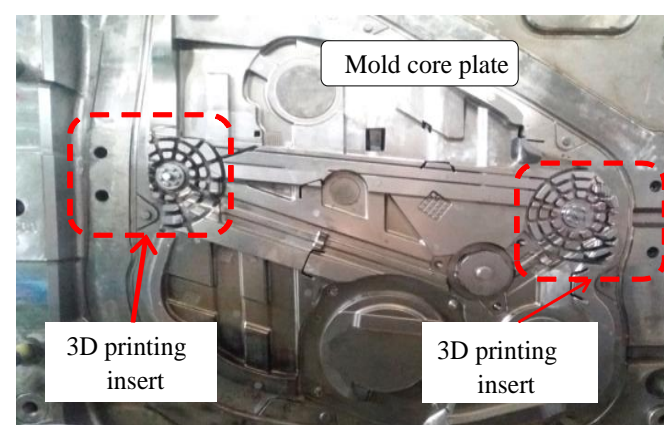

(c) Assembly to the mold

Fig. 5. Insert with conformal cooling channel inside was made by $3 \mathrm{D}$ printing technology 


\section{The smart mold system development}

During the molding process, there may be undesired change of process parameters and uncontrolled disturbance. These variations can result in inconsistence of product quality such as sink marks, short shot, flash, or large warpage. Therefore, the sensor system has be installed in the mold cavity in order to monitors, records, and controls temperature and pressure signals. Base these data, smart molding system integrated with the molding machine control system will be develop. The diagram of the smart mold system has been figured out as shown in figure 7. Process monitoring criteria are based on maximum value of pressure and temperature, minimum value of pressure and temperature, integral, average value, threshold, and window of pressure and temperature. The control algorithm for the smart mold system can be fuzzy logic, PID control and neural network.

There are three issues that will be focused on smart mold system:

(1) Control the temperature of barrel and hot runner:

We will control the temperature of barrel and the temperature of hot runner to obtain the desired plastic's meld temperature inside the mold.

(2) Control the injection molding machine:

To make the smart mold in order to assure the quality consistency of the molded part regardless of the change of injection molding machine or the variation of environment, the system must be integrated with the molding machine control system (control injection speed and packing pressure).

(3) Control the mold temperature:

Mold temperature is very important to the cooling time and the quality of the molded part. To control the mold temperature, we have to control the coolant (water). There are two ways:

a) Control the inlet water temperature: lower the inlet temperature can effectively lower the mold temperature.

b) Control the flow rate: increase the flow rate will cool the mold better.

Method (a) is has large control threshold (large margin), but it is expensive and more complex than method (b). Method (b) uses a servo motor to control the speed of coolant pump. This method simple than method (a) but the threshold is narrow. For example, we can only change the mold temperature around $2 \div 3^{\circ} \mathrm{C}$.

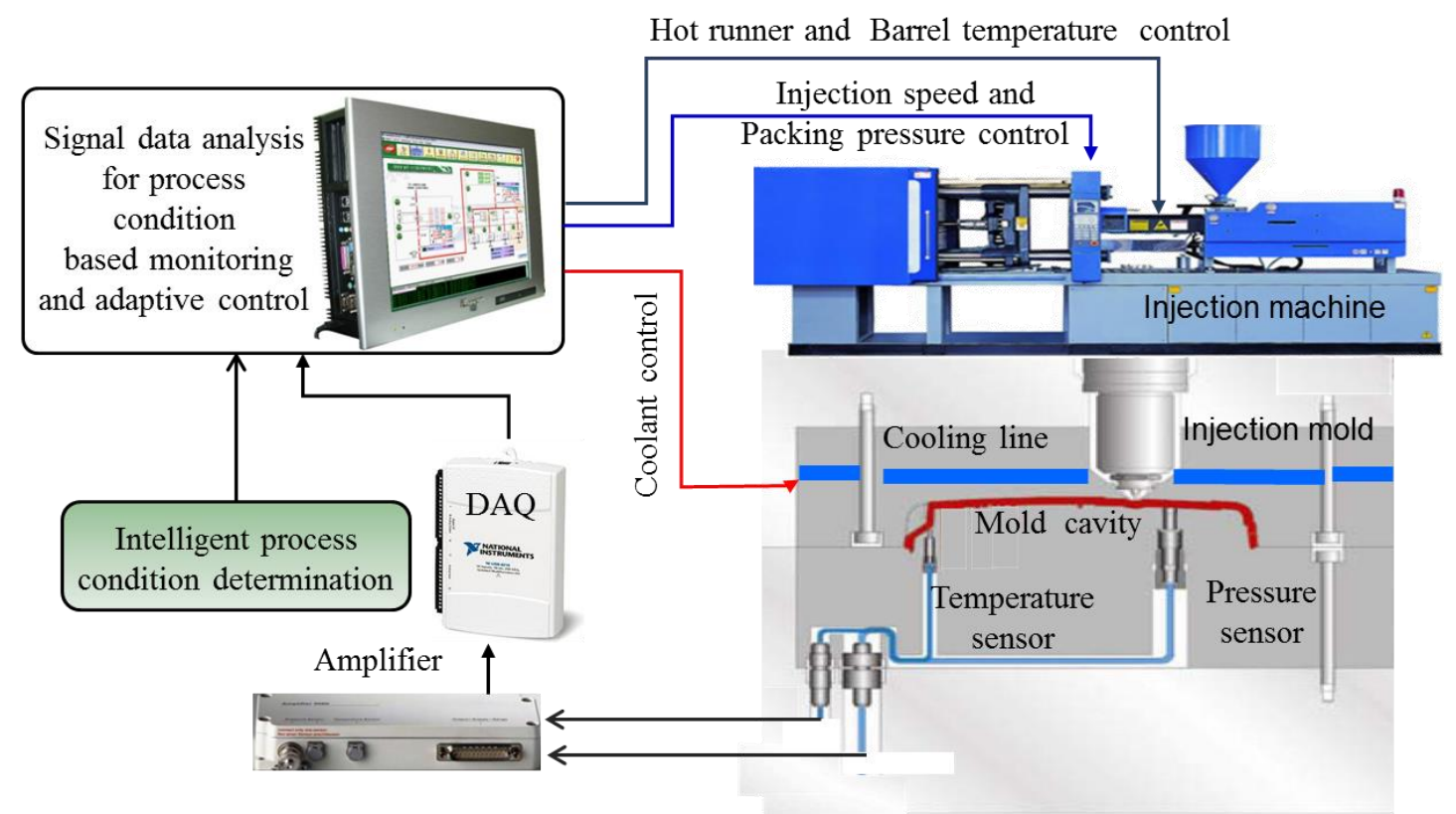

Fig. 6. The diagram of the smart mold system

\section{Research results and discussion}

After making the inserts, the testing process was done in order to verify the research results. The molding process was carried out on an industrial injection molding machine Toshiba IS2500DF with 2500 tons of clamping force. The result shows that's the real cooling time can be reduced from $39 \mathrm{sec}$ to $30 \mathrm{sec}$ (equivalent to $23 \%$ ) with the new cooling channel when using the coolant flow rate 2.25 litter/minute. There is error between simulation and real experiment due to the simulation error material properties. In addition, the microstructure and physical properties of 3D printing material is different from those of the same metal made by metallurgy. When using the conformal cooling channels, the appearance (a quality criterion) of the molded part also looks better than the one made with old cooling channels. The cycle time is 44 seconds. It is clear that the cooling time is reduced significantly. The error between simulation model and the real result is the limitation of this work. It is difficult to get the exact thermal conductivity data of material after $3 \mathrm{D}$ printing. I addition, the equivalent model is also an approximate approach. Therefore, error always occur. 


\section{Conclusion and future work}

This work studies on a conformal cooling channels applied in a medium-size injection mold that makes an automotive part. We improved the cooling system of an existent mold in order to reduce the cycle time and improve the quality of molded part. In injection molding, cooling stage is very important because it account for more than two thirds of the molding cycle. For that reason, we used spiral cooling channels made by 3D printing method. It is very expensive to make a large mold by 3D printing method; therefore, local approach was adopted. As the results, inserts with conformal cooling channels that is used to cool extremely hot locations in the mold is an intelligent solution.

The design of conformal cooling channels was done using the combination of analytic and CAE simulation method. Analytic method was used to roughly identify the cooling channel parameters and the simulation method was employed to check and fine-tune the design and visualize the results. We analysed the current state and performance of the existent mold so that its drawback was drawn. The literature review method was also used to approach the state of the art and to decide the optimal solution. The spiral cooling channel was selected as the best choice. Selective laser melting method was used to fabricate the inserts. The real testing results show that the cooling time can be reduced to $23 \%$. This is a significant improvement in the first phase of this project. In the next phase of this project, we develop the smart system for the mold as figured out in Section 4. The intelligent mold is still on the development process. By using the cavity sensor system on the second phase of our project, the temperature and pressure at considered (important) locations in the mold will be monitored; the intelligent molding control system will be developed and implemented based on the thermal and mechanical behaviour happen in the mold. The productivity and the quality of the molding process will be consistent regardless of disturbance and uncontrolled variations.

\section{Acknowledgement}

This work is supported by the Ministry of Trade, Industry \& Energy (MOTIE, Korea) under Industrial Technology Innovation Program. No. 10062677. "For plastic injection molds with 50\% improved cooling efficiency, development of $3 \mathrm{D}$ printing equipment $\&$ mold steel powder manufacturing technology having $30 \mu \mathrm{m}$ grade in diameter".

\section{References}

[1] Balc N. C.; Berce P. \& Pacurar R (2010). Comparison between SLM and SLS in producing complex metal parts, Annals of DAAAM for 2010, Vienna, Austria.

[2] Ferreira, J.C. \& Mateus A. (2003). Studies of rapid soft tooling with conformal cooling channels for plastic injection moulding, Journal of Materials Processing Technology, 142(2): pp. 508-516.

[3] Wang, Y.; Yu K.-M.; \& Wang C.C.L. (2015). Spiral and conformal cooling in plastic injection molding," ComputerAided Design, 63: pp. 1-11

[4] Park, H.S. \& Pham N.H. (2009). Design of conformal cooling channels for an automotive part, International Journal of Automotive Technology, 10(1): pp. 87-93.

[5] Au, K.M. \& Yu K.M. (2007). A scaffolding architecture for conformal cooling design in rapid plastic injection moulding, The International Journal of Advanced Manufacturing Technology, 34(5): pp. 496-515.

[6] Wang, Y., et al. (2011). Automatic design of conformal cooling circuits for rapid tooling, Computer-Aided Design, 43(8): pp. 1001-1010.

[7] Shayfull, Z., et al. (2014). Potential of Conformal Cooling Channels in Rapid Heat Cycle Molding: A Review," Advances in Polymer Technology, 33(1), 2014.

[8] Rahim, S.Z.A., et al. (2016). Improving the Quality and Productivity of Molded Parts with a New Design of Conformal Cooling Channels for the Injection Molding Process," Advances in Polymer Technology, 35(1).

[9] Jahan, S.A. and Mounayri H. El (2016). Optimal Conformal Cooling Channels in 3D Printed Dies for Plastic Injection Molding, Procedia Manufacturing, 5: pp. 888-900.

[10] Ahn, D.-G., Park, S.-H. \& Kim H.-S. (2010). Manufacture of an injection mould with rapid and uniform cooling characteristics for the fan parts using a DMT process, International Journal of Precision Engineering and Manufacturing, 11(6): pp. 915-924.

[11] Khan, M., et al. (2014). Cycle Time Reduction in Injection Molding Process by Selection of Robust Cooling Channel Design, ISRN Mechanical Engineering, 2014: pp. 8.

[12] Rännar, L.E.; Glad A.; \& Gustafson C.G. (2007). Efficient cooling with tool inserts manufactured by electron beam melting, Rapid Prototyping Journal, 13(3): pp. 128-135.

[13] Park H. \& Pham N. (2008). A new approach to conformal cooling channels in injection molding process. DAAAM International Scientific Book 2008, pp. 587-602

[14] Miyamoto, S. \& Yukutomo H. (2011). Smart Molding Simplifies Molding Jobs - New Si-V Series Electric Injection Molding Machines, Hitachi Review, 60(5).

[15] Chrystal, S. (2013). Ensuring Injection Molded Part Consistency and Conformance through In-Mold Cavity Sensor Technology, in Precision Engineered Products 2013.

[16] Dang, X.-P. \& H.-S. Park (2011), Design of U-shape milled groove conformal cooling channels for plastic injection mold, International Journal of Precision Engineering and Manufacturing, 12(1): pp. 73-84. 